\title{
MULTIOBJECTIVE OPTIMIZATION OF HARD DISK SUSPENSION ASSEMBLIES: PART II-INTEGRATED STRUCTURE AND CONTROL DESIGN
}

\author{
Yee-Pien Yang and Yi-An Chen \\ Department of Mechanical Engineering, National Taiwan University, Taipei, Taiwan 106, \\ Republic of China
}

(Received 23 September 1994)

\begin{abstract}
The design of activcly controlled hard disk suspension assemblics is formulated as a multiobjective optimization problem. The integrated structure/control objectives consist of natural frequencies and an optimal control performance index with weighted system state regulation errors and control efforts, subject to some side constraints on design variables that describe the geometry of the suspension. Two multiobjective optimization techniques, goal programming and compromise programming, are implemented through an interface program communicating with an advanced finite element analysis program. The feasibility of the optimal design is demonstrated and the final decision making is also discussed.
\end{abstract}

\section{INTRODUCTION}

The flying height of the slider over the rotating disk always dominates the record density, hence the size, weight and capacity of a hard disk drive. The fluctuation of the flying height is inevitable during the disk operation, stemming from disturbances such as vibrations of actuator arm, rotating flow induced vibrations, lateral positioning motion induced out-ofplane vibrations, inaccuracies of spindle alignment, and so on. Either passive design or active control on the suspension assembly can be used to reduce the suspension vibration, thereby keeping the flying height as small as possible. However, better structural designs usually facilitate the implementation of active vibration controls, which would have less effort than controlling a structure without optimal designs. Moreover, the inclusion of the control performance in the structural optimization must result in more feasible designs. This motivates many researchers devoting themselves to the integrated structure and control optimization technique, and this has received tremendous attention in recent years, due to the increasing demands on the reduction of structure weight and control effort, and on the improvement of closed-loop system response. Several approaches are being developed and used in the design and control optimization problems, and these can be broadly classified as: (1) sequential optimization; (2) simultaneous optimization; and (3) multiobjective optimization and other optimization techniques.

The sequential optimization technique carries out first the structural (or control) design followed sequentially by the control (or structural) design.
This process is repeated iteratively until the optimal solution is found to satisfy certain convergence criteria. One of the applications of this technique was addressed by Venkayya and Tischler [1]. The structural optimization was posed as the minimization of structural mass with the constraint on the fundamental frequency, and subsequently minimized was a quadratic performance function of the the energy of actuators and structural vibrations. The drawback of this approach is that the integrated solution depends on the sequential ordering of the control and structure design solutions.

The simultaneous optimization technique allows the designer to formulate a performance index, in structural parameters and control variables, as the sum of structural properties, such as mass or fundamental frequency, and the quadratic performance index associated with a linear regulator optimal control problem. Recently, Grandhi [2] presented a simultaneous structure and control optimization of flexible structures. The structural weight or the Forbenious norm was minimized with constraints imposed on the closed-loop eigenvalues as well as damping parameters. Onoda and Watanabe [3] integrated the optimization of structure with the LQG controller, taking into account the suppression of the residual modes. The cost functional consisted of control effort and normalized structural mass, as well as weighting factors to guarantee a certain amount of modal damping ratios. Slater and McLaren [4] dealt with an integrated optimization of structure and control of flexible spacecrafts. Their design was to find structural parameters and control law to minimize a performance index that was system weight 
while satisfying control energy and displacement constraints. Other examples of simultaneous optimization includes Bodden and Junkins [5], Hale et al. [6], Horta et al. [7], Miller and Shin [8], Lust and Schmit [9], Thomas et al. [10], and so on. With this method, the sensitivity analysis may be of high order since the objective and constraint functionals must include both the control system and the structure with various design variables and weightings. Usually there exists no unique solution that would give the optimum for all objective functions simultaneously.

Various multiobjective optimization techniques have been proposed and applied in the industry, as surveyed by Tseng and Lu [11]. For the application to the combined structure/control optimization, Rao et al. [12] used the cooperative game theory for the design of actively controlled structures subject to the constraints on damping parameters of the closedloop system, formulating a multiobjective optimization problem. The structural weight and control energy were objective functionals for minimization with cross-sectional areas of members as design variables. Livine et al. [13] formulated the synthesis of an actively controlled composite wing as a multidisciplinary optimization problem, where a unique integration of analysis techniques spanning the diciplines of structures, aerodynamics, and controls is described. Gilbert and Schmidt [14] proposed a multilevel optimization approach to the integrated structure/control law design. The lower level consisted of independent structural design and control law design, and the design results and sensitivities were coordinated through the upper level optimization problem that reflected the desired objectives of the integrated structure/control law design.

With a sequential process in each optimization loop, Bruch et al. [15] proposed an alternative, namely, quasi-simultaneously multiobjective optimization approach to the simultaneous structural design and active control of a symmetric, cross-ply laminate. The problem was formulated as a multiobjective optimization problem with a two weighted sum of design and control objectives as performance indices, which consisted of common objectives on maximizing the fundamental frequency and minimizing dynamic responses, while one of which differs from the other with an additional objective on the minimization of either the closed-loop or open-loop control effort. Two performance indices were minimized simultaneously. However, the performance index with the additional control effort was minimized with respect to control gains, while using the optimal structural design variables obtained by minimizing the other performance index with respect to the structural design variables.

The goal of Part II is to integrate structure and control optimization techniques on the shape design of suspension assemblies of hard disks. The design objective is to raise natural frequencies of the suspension assembly so that it will not be excited easily by undesirable disturbances, as stated in Part I. Simultaneously, the state regulation errors and vibration control efforts are minimized with respect to structural parameters as well as control gains.

\section{OPTIMAL CONTROL OF PARAMETRIC EQUATIONS}

\subsection{Modal analysis}

In this section a generic class of optimization problem is defined specifically for vibration control of flexible structures. In terms of design variables $x$, the $N$ th-order equations of motion (1) that describe the dynamic behavior of suspension assembly can bc transformed to principal (modal) coordinates

$$
\ddot{\eta}+\mathbf{C}^{\prime}(x) \dot{\eta}+\mathbf{K}^{\prime}(x) \eta=\mathbf{B}^{\prime}(x) u
$$

by the matrix transformation

$$
\mathbf{q}=\boldsymbol{\Phi}(x) \boldsymbol{\eta}
$$

where $\boldsymbol{\eta}$ is the modal coordinate vector, $\boldsymbol{\Phi}(x)$ is the modal matrix whose columns are the corresponding normal modes, that is

$$
\boldsymbol{\Phi}(x)=\left[\phi_{1}, \phi_{2}, \ldots, \phi_{N}\right]
$$

For simplicity, the argument $x$ is omitted for subsequent analyses. The matrices $K^{\prime}, \mathbf{C}^{\prime}$ and $\mathbf{B}^{\prime}$ have been normalized, so that we call the modal stiffness, modal damping, and modal input influence matrices, respectively, given by

$$
\begin{gathered}
\mathbf{K}^{\prime}=[m]^{-1} \boldsymbol{\Phi}^{T} \mathbf{K} \boldsymbol{\Phi}=\operatorname{diag}\left(\omega_{1}^{2} \omega_{2}^{2} \ldots \omega_{N}^{2}\right) \\
\mathbf{C}^{\prime}=[m]^{-1} \boldsymbol{\Phi}^{T} \mathbf{C} \boldsymbol{\Phi}=\operatorname{diag}\left(2 \zeta_{1} \omega_{1} 2 \zeta_{2} \omega_{2} \ldots 2 \zeta_{N} \omega_{N}\right) \\
\mathbf{B}^{\prime}=[m]^{-1} \boldsymbol{\Phi}^{T} \mathbf{B}
\end{gathered}
$$

in which

$$
[m]=\boldsymbol{\Phi}^{T} \mathbf{M} \boldsymbol{\Phi}=\operatorname{diag}\left(m_{1} m_{2} \ldots m_{N}\right)
$$

is a diagonal modal mass matrix, $\zeta_{i}$ and $\omega_{i}$ are the damping ratio and natural frequency of the $i$ th normal mode.

\subsection{Optimal control formulation}

By the modal analysis the transformation to principal coordinates has uncoupled the equations of motion, leading to $N$ separate single-degrees-offreedom equations. In fact, high-frequency modes possess less kinetic and potential energy, and decay much faster than low-frequency modes due to the structural damping. It is efficient and practical for the designer to truncate those modal coordinates that correspond to high-frequency modes. In the following optimal control formulation, selected are $S$ modal coordinates that describe the dominant dynamic be- 
havior of the suspension assembly. In the state-space form, eqn (1) is expressed by

$$
\dot{\mathbf{y}}=\mathbf{A y}+\mathbf{B} u
$$

where $\mathbf{y}=\left[\eta^{T} \dot{\eta}^{T}\right]^{T}$ is the state variable vector, and $\mathbf{A}$ and $\mathbf{B}$ are the plant and input matrices given by

$$
\mathbf{A}=\left[\begin{array}{cc}
0 & I \\
-\mathbf{K}^{\prime} & -\mathbf{C}^{\prime}
\end{array}\right] \text { and } \mathbf{B}=\left[\begin{array}{c}
0 \\
\mathbf{B}^{\prime}
\end{array}\right]
$$

In order to design a linear quadratic regulator a performance index $(P I)$ can be defined as

$$
f_{4}=\frac{1}{2} \int_{0}^{\infty}\left(\overline{\mathbf{q}}^{T} \mathbf{Q} \overline{\mathbf{q}}+\boldsymbol{u}^{T} \mathbf{R} u\right) \mathrm{d} t,
$$

where $\overline{\mathbf{q}}=\left[\mathbf{q}^{T} \dot{\mathbf{q}}^{T}\right]^{T}$, and $\mathbf{Q}$ and $\mathbf{R}$ are the state and control weighting matrices which have to be positive semi-definite and positive definite, respectively. Suppose that the system is either uniformly completely controllable or exponentially stable, the minimization of the performance index for a set of design variables yields the steady-state optimal control law

$$
u^{*}=-\mathbf{R}^{-1} \mathbf{B}^{\mathrm{T}} \mathbf{P y}
$$

where $\mathbf{P}$ is the Riccati matrix that satisfies the algebraic equation

$$
\mathbf{A}^{T} \mathbf{P}+\mathbf{P A}-\mathbf{P B R}^{-1} \mathbf{B}^{T} \mathbf{P}+\Phi_{d}^{T} \mathbf{Q} \Phi_{d}=0
$$

in which $\Phi_{d}=\operatorname{diag}(\Phi \Phi)$. Therefore, the governing equation of the optimum closed-loop system can be written as

$$
\dot{\mathbf{y}}=\overline{\mathbf{A}} \mathbf{y},
$$

where

$$
\overline{\mathbf{A}}=\mathbf{A}-\mathbf{B R}^{-1} \mathbf{B}^{T} \mathbf{P} .
$$

\subsection{Dynamic responses}

The above optimal control formulation can be used in two ways. First, the optimal control analysis is performed after the structure optimization is completed; that is, the optimal shape of the suspension assembly is determined by minimizing the objective functions $f_{1}, f_{2}$ and $f_{3}$ defined in eqns (2)-(4) of Part $I$, and then the control responses are examined. On the other words, the optimization will be carried out independently with dual sets of objective functions. Second, all the objective functions $f_{i}, i=1-4$ are considered simultaneously in the multiobjective optimization techniques: goal programing and compromise programming, as stated in Appendix B of Part I. The second method will be elaborated in the next section.

Disturbance responses. Since the major vibrations of the suspension assembly are exerted by the disturb- ances from either the air-bearing fluctuations or the rotating flow between disks, the disturbance force distribution depends on the preload of the suspension, flying height, track location of the slider, rotation speed of the disk, and so on. The displacement response is investigated at the slider head with respect to disturbance force inputs at three different locations on the suspension assembly, as shown in Fig. 3 of Part I. The transfer functions between these disturbance inputs (forces) and the slider head output (displacement) are first computed by selecting the first six modes of the original suspension assembly, whose dimensions are listed in Appendix A of Part I.

Figure 1 shows the frequency responses of the slider head displacement with respect to the three input points of disturbances. It is apparent that the disturbance input point $a$, near the centroid of the suspension beam, produces the most oscillation on the slider head, while the disturbance input at point $c$ on the slider only slightly excites the head because of the large stiffness of the air bearing. The phase delay due to the wave propagation from the disturbance input to the output point can be observed in the phase plot. Moreover, a small amount of change in the magnitude produces a much larger change in the phase of plot $c$ than the other two phase plots. This is due to the noncolocation of input and output of a flexible structure, thereby resulting in a nonminimum phase transfer function.

Optimal control responses. For a better control performance with less control effort, two input points are selected at $a$ and $b$ for simulation. It is true that the symmetric torsional modes are uncontrollable if the inputs act on the nodal lines of the suspension. However, the fundamental mode is usually a bending mode whose magnitude is much larger than that of high-frequency modes, and is a major part of vibration to suppress.

As the optimal control strategy is performed, the weighting matrices $R$ and $\mathbf{Q}$ are chosen so that $R=1$ and all elements of $\mathbf{Q}$ are zeros except $\mathbf{Q}_{k k}=10^{15}$, the diagonal element corresponding to the output displacement point $O$. By substituting the optimal control $u^{*}$ of eqn (11) into eqn (1), and calculating the closed-loop transfer functions for output point $O$ with respect to input points $a$ and $b$, we obtain their frequency responses as shown in Fig. 2. The input points $a$ and $b$ are, respectively, close to the maximum magnitudes of the first (bending) mode and the second bending (the fourth) mode. It is not surprising that the magnitudes of the first two bending modes (the first and fourth modes) are effectively reduced, in comparison with the frequency response of the uncontrolled system that the dashed curve describes. Moreover, time responses of the optimal control performance compared with the uncontrolled (openloop) response are presented in Fig. 3, associated with its optimal control effort. Note that for the regulation control of the suspension the initial displacement of 

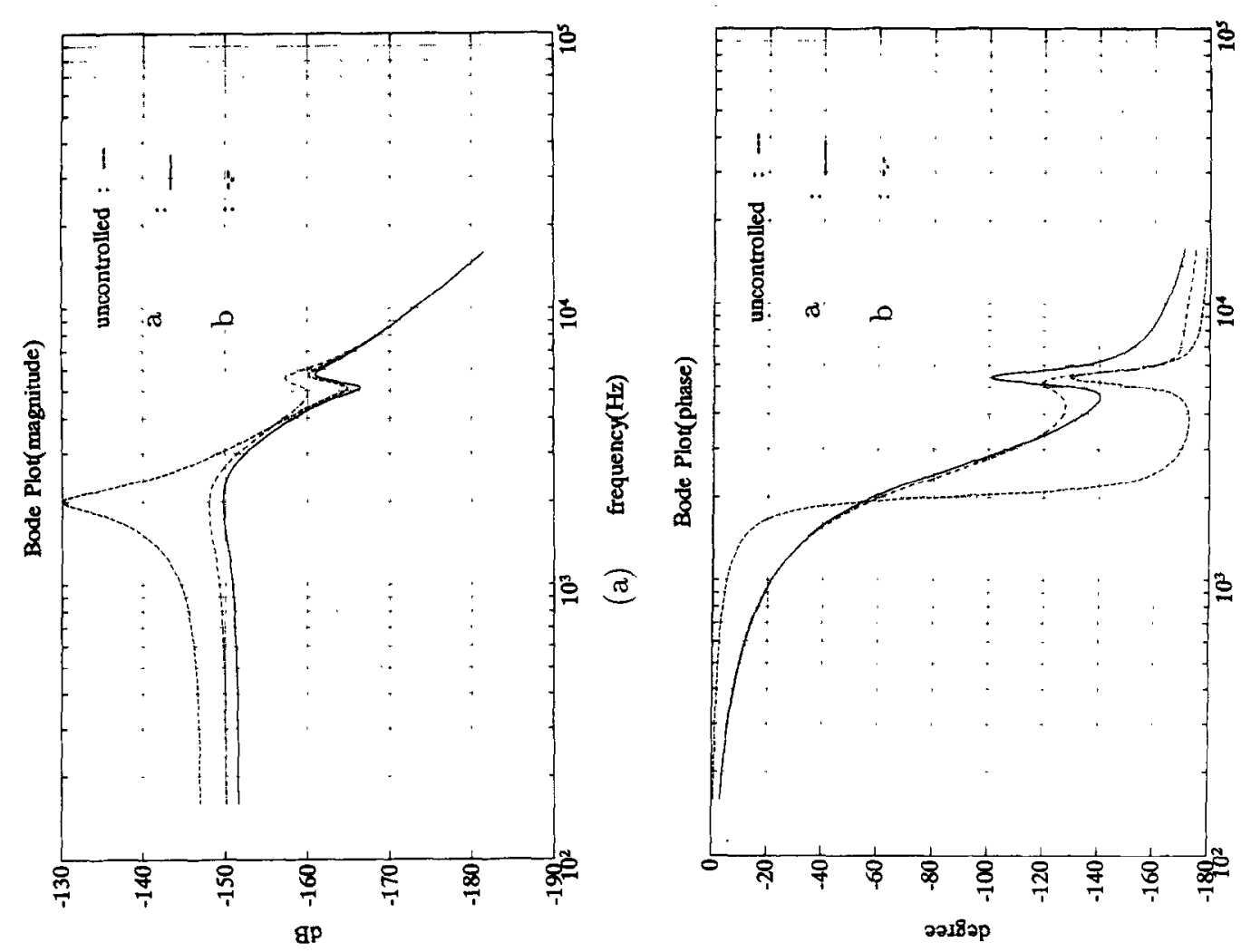

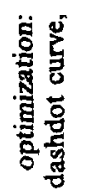

范 동류 공

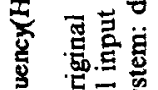
莺 爮峞

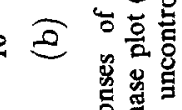
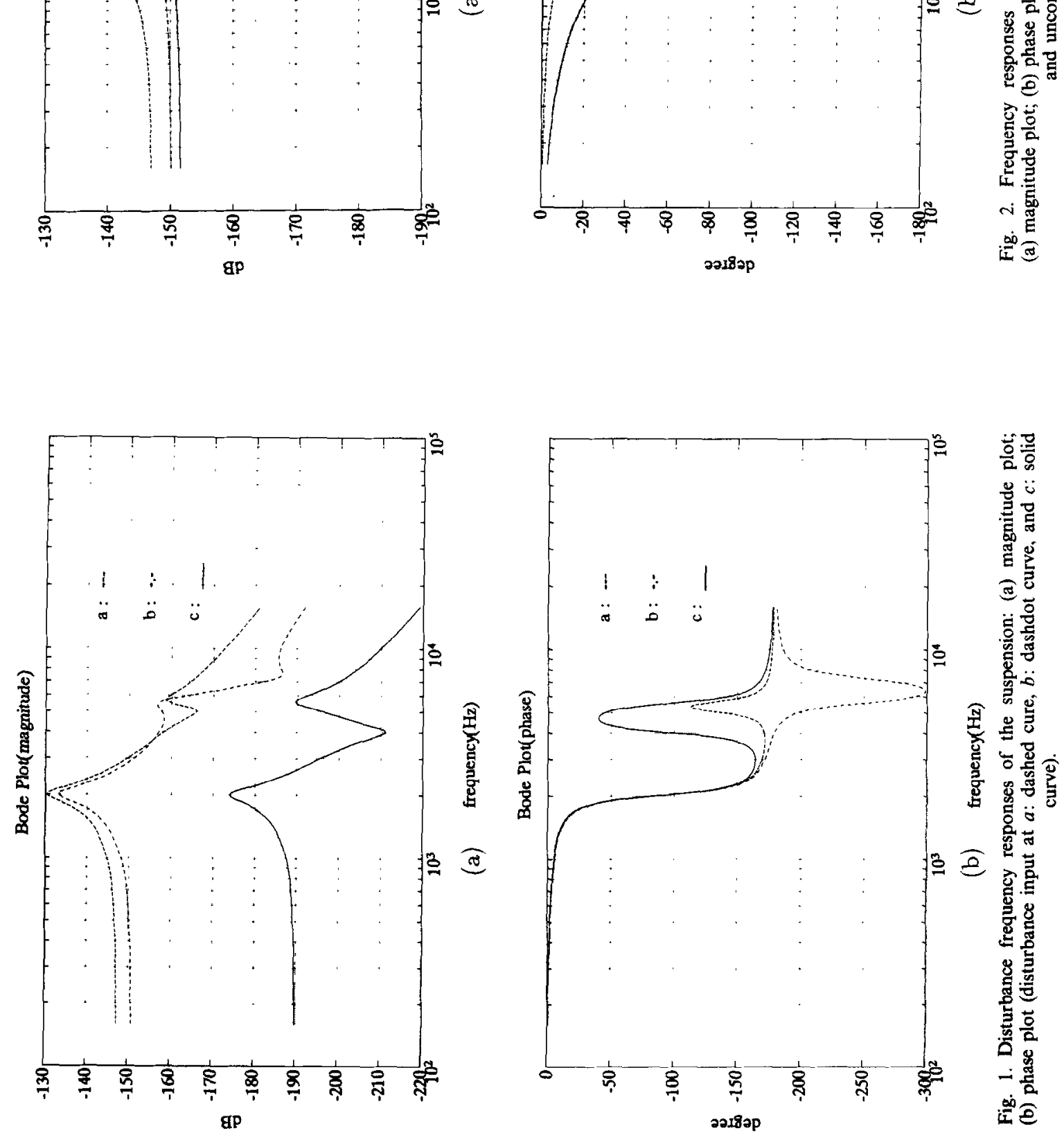

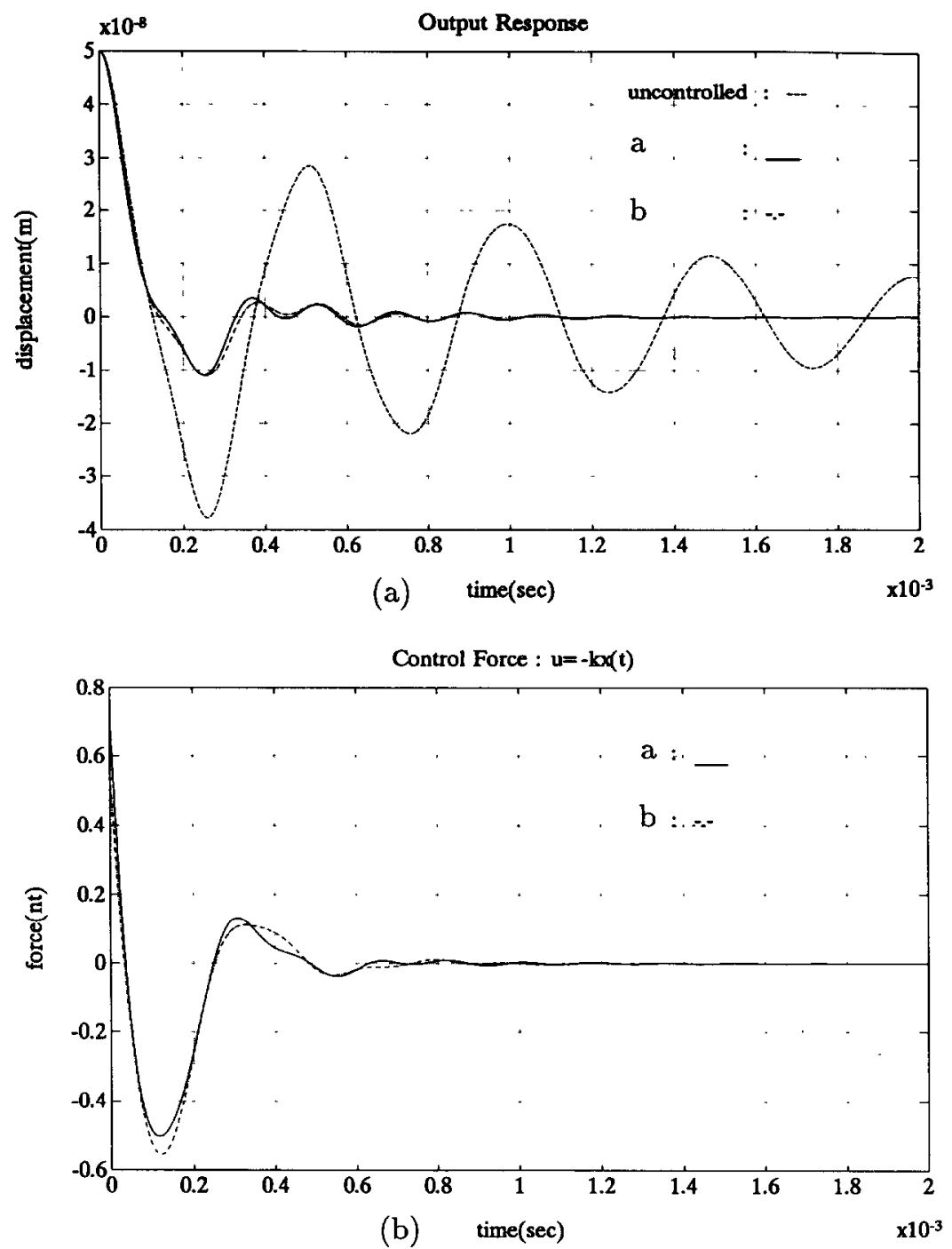

Fig. 3. Open and closed-loop time responses of the original suspension without optimization: (a) displacement; (b) control force (control input at $a$ : solid curve, $b$ : dashdot curve, and uncontrolled system: dashed curve).

the slider head is given as $0.05 \mu \mathrm{m}$, around $15 \sim 25 \%$ of the usual flying height $0.2 \sim 0.3 \mu \mathrm{m}$.

\section{INTEGRATED OPTIMAL STRUCTURE AND CONTROL DESIGN}

\subsection{Multifunctional objectives}

As in Part I, the shape of the suspension assembly is designed at its loaded status. Both objectives, to keep away from the excitation of disturbances and to actively suppress undesirable vibrations, motivate the designer to choose additional cost function $f_{4}$ of eqn (10) along with the previous cost functions $f_{1}, f_{2}$ and $f_{3}$ of eqns (2)-(4) of Part I, subject to the side constraints listed in Table 1 of Part I. The minimum and maximum values of $f_{4}$ are calculated as 1.6943 $\times 10^{-4}$ and $7.6234 \times 10^{-4}$, respectively.

\subsection{Computational flow}

As in Part I two multifunctional optimization techniques are investigated-goal programming and compromise programming. The major steps involved in the structure/control optimization are as follows:

(1) Read the MOST input file All the input data are divided into three blocks, problem identification and initial parameters, design variable data, and multiobjective optimization methods and their parameters.

(2) Start the master program

Initially, the values of design variables are given, and the master program MOST will first evaluate the objective and constraint functions by invoking ABAQUS for structure analysis. Then the gradients of objectives and constraints are calculated for 
searching a descent direction and a step size to obtain a new design point. In that way, MOST will branch into one of the three possible subroutines:

(2.1) Evaluation of objective and constraint functions

To proceed in this part, the finite element analysis must be performed. As ABAQUS is invoked, four steps are taken for structure analysis:

(a) Mesh generation-this is accomplished by a subroutine which generates coordinates of the structure to be analyzed, subject to the change of design variables.

(b) Preparation of ABAQUS input file - a specific format of data input must be provided by the user for the problem to be solved by ABAQUS.

(c) Execution of ABAQUS - this step starts at the moment when a child process is forked. As the structure analysis with ABAQUS is being executed in the child process, the MOST program is sleeping until the complete analysis succeeds within an assigned sleeping time.

(d) Read results file-the results file of ABAQUS saves variables in a specific format of binary or ascii code. The master program in MOST must identify and grasp what are required for subsequent analysis, e.g. natural frequencies and their corresponding modes.

(e) Evaluation of optimal control cost--a set of linear state-space equations of the structure control problem are formulated by choosing a finite number of effective modes according to the above analysis. Then the optimal control law, hence the optimal control cost, can be determined by Riccati equations.

The objective and constraint functions are then subsequently evaluated from the results of the structural analysis.

(2.2) Gradient evaluation

Two options for gradient evaluation are available in MOST:

(a) User-provided gradient - the user can program gradient expressions for the objective and constraint functions in separate subroutines. The gradient expressions can also be verified by checking the gradient evaluation by the finite difference approach.

(b) Finite difference method-the forward, backward, or central finite difference method can be specified for calculating the gradients if users do not program the gradient expressions.

During the gradient evaluation with the finite difference approach, the optimizer will repeat the evaluation of the objective and constraint functions with perturbed design variables of specified variations by invoking ABAQUS repeatedly.

(2.3) Convergence analysis

Various convergence criteria of the optimization algorithm are specified in MOST:

acv-acceptable violation of constraints for feasible designs. For example, if acceptable tolerance for maximum constraint violation for feasible design is $0.01 \%$ then $a c v$ is set to 0.0001 .

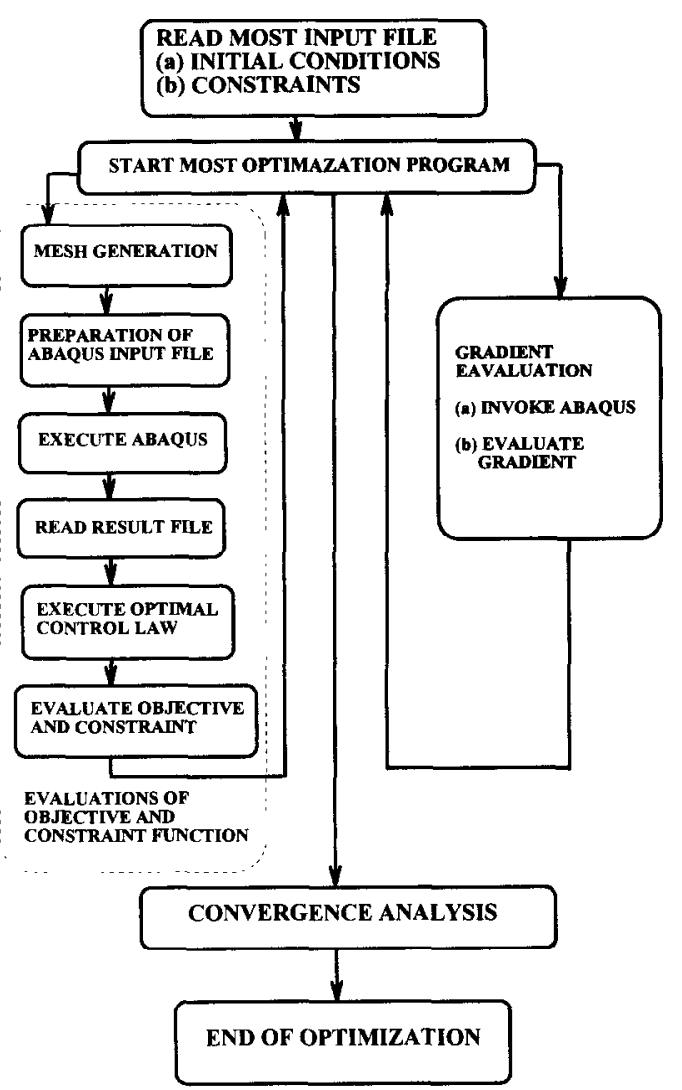

Fig. 4. Computation flow of structure/control optimization.

acs-acceptable tolerance for the convergence parameter for optimum solution. The convergence parameter should be zero at the optimum. However, numerically, a value of acs between 0.001 and 0.0001 has worked fairly well.

Values of $a c v$ and acs control final convergence of the optimization algorithm. Smaller values of these parameters will generally take more iterations to satisfy the convergence criteria.

The computational flow of the optimization, along with the structural analysis and the optimal control, is illustrated in Fig. 4.

\subsection{Optimization results}

For comparison with the results obtained in Part I, the same weightings of the two multifunctional optimization techniques are used. Both the indices $\beta$ and $\gamma$ are chosen as 1 and 2 for goal and compromise programmings, respectively. The optimization results are shown in Tables 1 and 2, and the optimal shapes in the finite element mesh for the cases of $\beta=1$ and $y=1$ are illustrated in Fig. 5 .

(1) The goal programming technique yields a wider base $y d$ of the suspension than the compromise programming, while results in shorter mounting length of the suspension $x d$. Therefore, the shape designed by the compromise programming technique looks a little slender. 


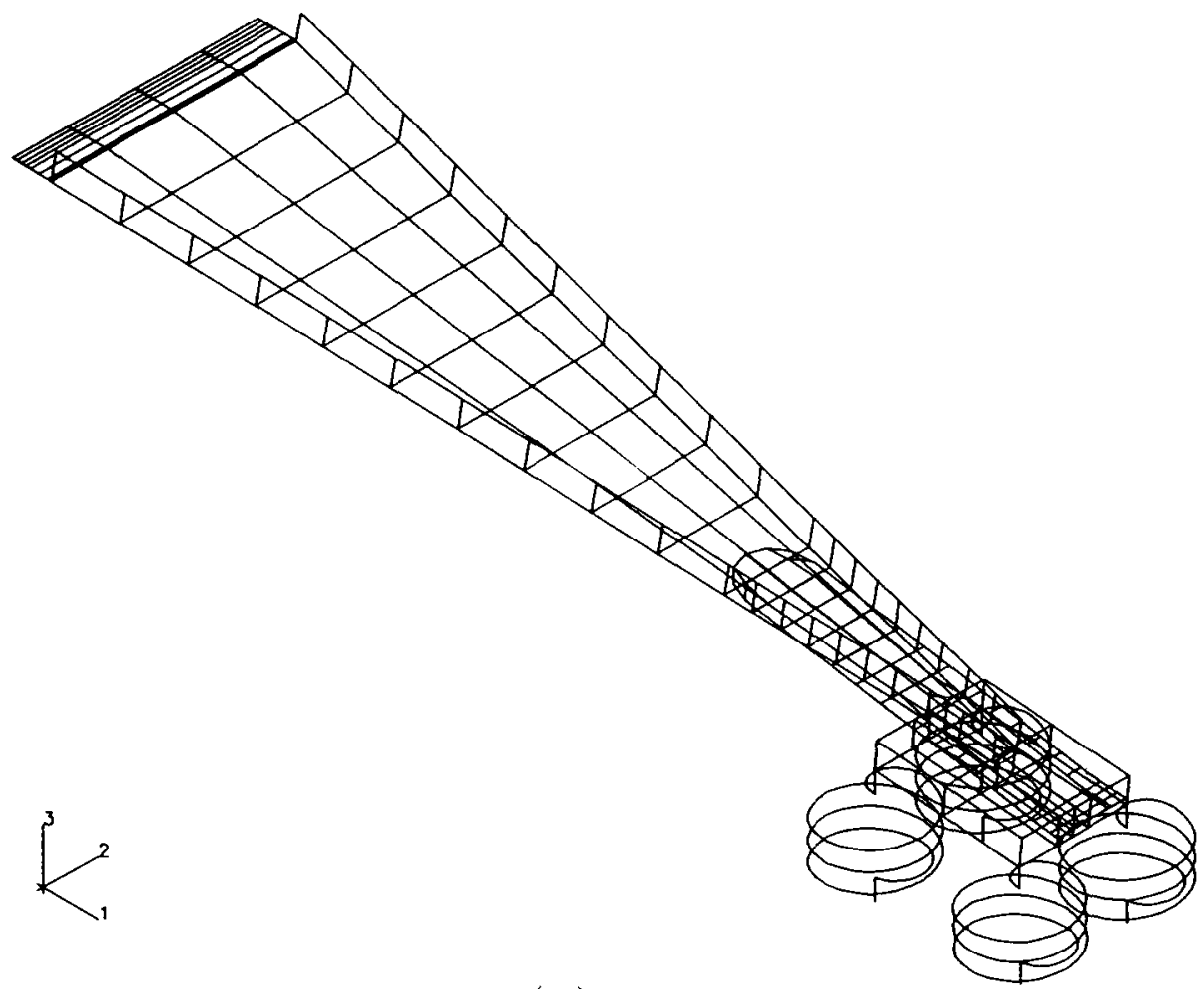

(a)

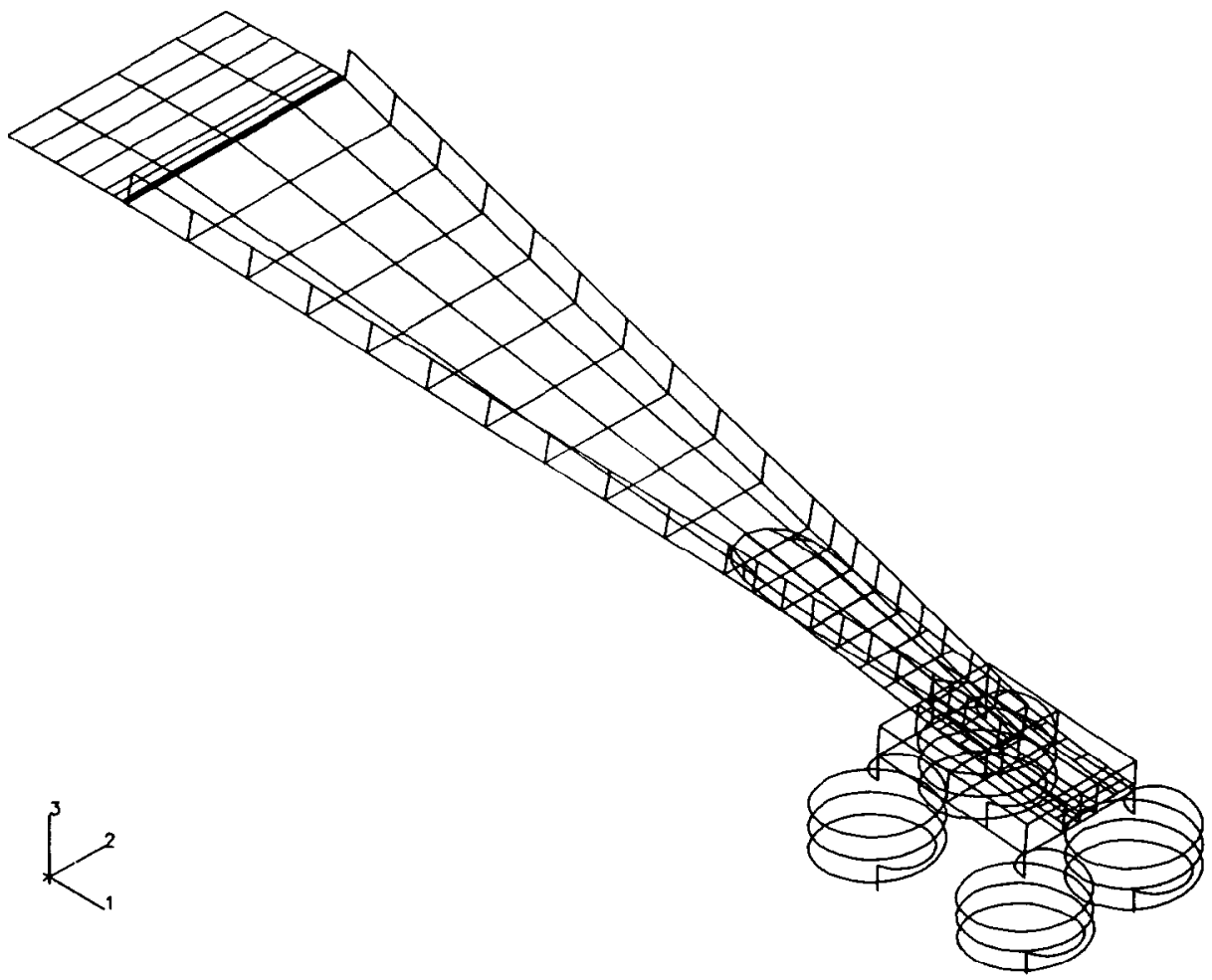

(b)

Fig. 5. Optimal structure/control design results: (a) goal programming $(\beta=1)$; (b) compromise programming $(\gamma=1)$. 
Table 1. Structure/control optimization design results with goal programming

\begin{tabular}{ccc}
\hline & $\beta=1$ & $\beta=2$ \\
\hline Design variable (mm) & & \\
$y b$ & 6.8729 & 6.8509 \\
$x d$ & 0.5000 & 0.5000 \\
$x 2$ & 0.1000 & 0.2890 \\
$w h$ & 0.7922 & 0.8000 \\
Objectives & & \\
$f_{1}$ & $4.0117 \times 10^{-4}$ & $3.9808 \times 10^{-4}$ \\
$f_{2}$ & $3.9364 \times 10^{-4}$ & $3.8969 \times 10^{-4}$ \\
$f_{3}$ & $2.9614 \times 10^{-4}$ & $2.9570 \times 10^{-4}$ \\
$f_{4}$ & $1.9497 \times 10^{-4}$ & $2.0562 \times 10^{-4}$ \\
Errors & & \\
$d_{1}^{+}$ & $0.4172 \times 10^{-4}$ & $0.3863 \times 10^{-4}$ \\
$d_{2}^{+}$ & $1.3695 \times 10^{-4}$ & $1.3300 \times 10^{-4}$ \\
$d_{3}^{+}$ & $0.6946 \times 10^{-4}$ & $0.6902 \times 10^{-4}$ \\
$d_{4}^{+}$ & $0.2554 \times 10^{-4}$ & $0.3619 \times 10^{-4}$ \\
Frequencies $(\mathrm{Hz})$ & & \\
$\omega_{1}$ & 2493 & 2512 \\
$\omega_{2}$ & 2540 & 2566 \\
$\omega_{3}$ & 5917 & 5948 \\
\hline
\end{tabular}

Table 2. Structure/control optimization design results with compromise programming

\begin{tabular}{ccc}
\hline$(\alpha=1)$ & $\gamma=1$ & $\gamma=2$ \\
\hline Design variable $(\mathrm{mm})$ & & \\
$y b$ & 6.0216 & 5.9600 \\
$x d$ & 2.6373 & 2.8549 \\
$x 2$ & 0.1000 & 0.2835 \\
$w h$ & 0.8000 & 0.8000 \\
Objectives & & \\
$f_{1}$ & $3.8084 \times 10^{-4}$ & $3.9119 \times 10^{-4}$ \\
$f_{2}$ & $3.4885 \times 10^{-4}$ & $3.4209 \times 10^{-4}$ \\
$f_{3}$ & $3.9303 \times 10^{-4}$ & $3.9934 \times 10^{-4}$ \\
$f_{4}$ & $2.0224 \times 10^{-4}$ & $2.0178 \times 10^{-4}$ \\
Frequencies $(\mathrm{Hz})$ & & \\
$\omega_{1}$ & 2626 & 2556 \\
$\omega_{2}$ & 2867 & 2923 \\
$\omega_{3}$ & 5411 & 5427 \\
\hline
\end{tabular}

(2) All the flap heights in the optimal design have a maximum allowable value $0.8 \mathrm{~mm}$, except for the case $\beta=1$ of the goal programming to get a little smaller value $0.7922 \mathrm{~mm}$. It is still hard to tell which case is better, the designer has to make a final decision with additional dynamical and manufacturing requirements on the suspension assembly.

(3) As in Part I, the final results in raising the natural frequencies are satisfactory. Without changing the original length, thickness and tip width of the suspension beam, the first and second natural frequencies are raised over $400-500 \mathrm{~Hz}$, and the difference between the second and the third natural frequencies increases at least $500 \mathrm{~Hz}$.

(4) The final value of each objective function, as shown in Table 1 and 2, is larger than its minimum value and less than its maximum value. It is obvious that the nondominated solutions, or superior solutions, are not achieved, but a set of optimal solutions are obtained by making compromise between objective functions with each other.

\section{DECISION MAKING}

In the above analyses, we have more than one alternative of optimal designs. The decision maker needs to select the most desirable alternative, and his rational choice requires a criterion by which he evaluates different alternatives and places them in some form of ranking. Back to the original design objectives, the natural frequencies of the suspension assembly have to be raised so that it will not be excited easily by undesirable disturbances. A little different from Part I, we investigate the closed-loop frequency responses of the optimal suspension shape with optimal controls. The single input point is selected at $a$, and the output is chosen at $O$ where the slider head displacement is measured, as shown in
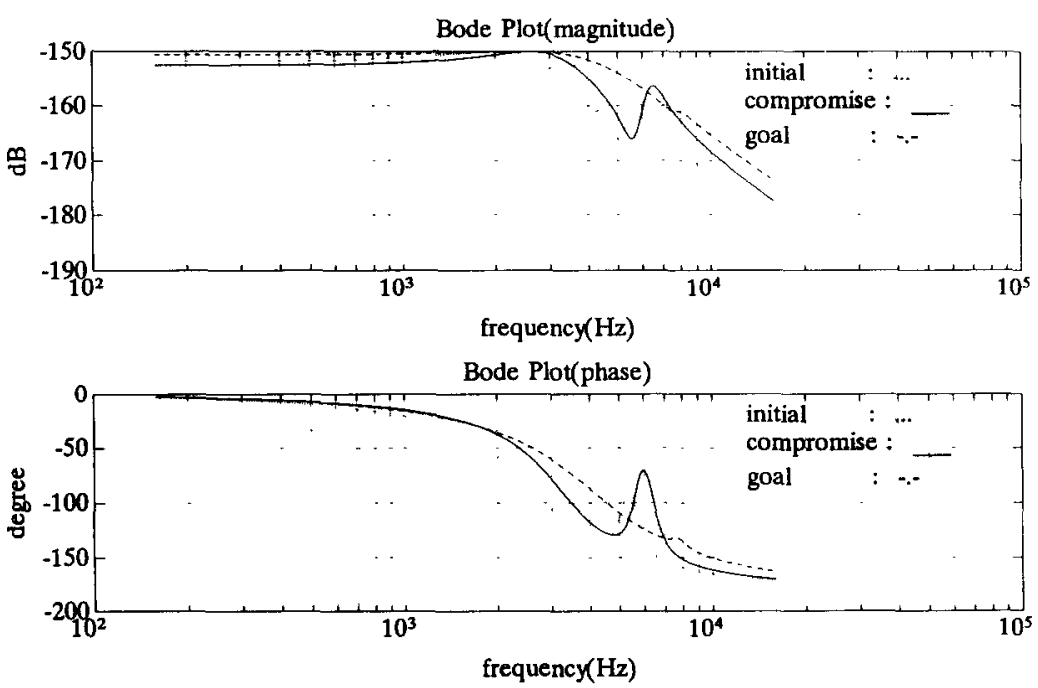

Fig. 6. Closed-loop frequency responses of the suspension with integrated structure/control design (solid curve: compromise programming, dashdot curve: goal programming, dotted curve: original suspension with optimal control) 

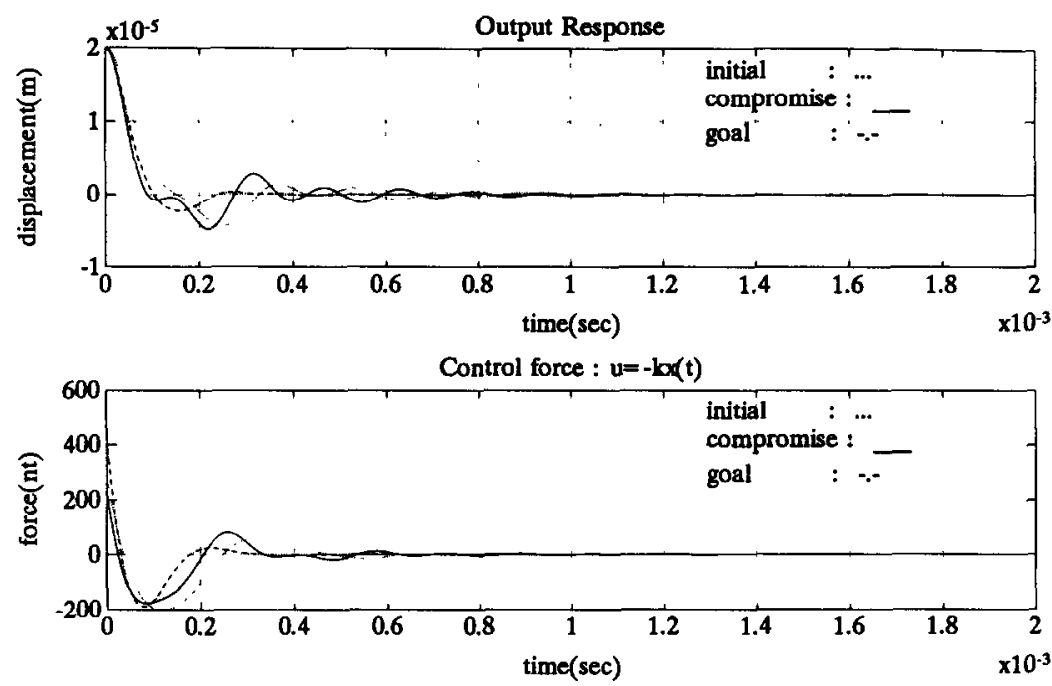

Fig. 7. Optimal control time responses of the suspension with integrated structure/control design (solid curve: compromise programming, dashdot curve: goal programming, dotted curve: original suspension with optimal control).

Fig. 3 of Part I. That way the symmetrical torsional modes are uncontrollable but observable. This will not cause system instability because the torsional modes are seldom excited by out-of-plane disturbances that produce flying height fluctuations, and those high-frequency torsional modes will decay fast due to the structural damping.

Figure 6 shows the closed-loop frequency responses of the suspension designed by goal programming $(\beta=1)$ and compromise programming $(\gamma=1)$, respectively, compared to the frequency response of the original suspension with an optimal control. It is interesting to find out that the low-frequency gain of the design with compromise programming is the smallest, while that with goal programming it is the largest. This indicates that low-frequency disturbances may bring about less fluctuation of the slider head with the compromise programming design than the other two designs. Moreover, the frequency response with the compromise programing design has more attenuation for higher frequencies, and a little larger bandwidth than that of the goal programing design.

In the time responses as shown in Fig. 7 though, an alternative decision may be made for the final solution. For the goal programming design, the rise time may be a little larger due to a smaller bandwidth, however its smaller resonant peak in frequency relates a smaller peak overshoot in the time response and a larger phase margin to guarantee the stability [16]. In fact, the settling time of the initial displacement response of the suspension design with goal programming is much smaller than the other two cases. This must be very critical since the vibration of

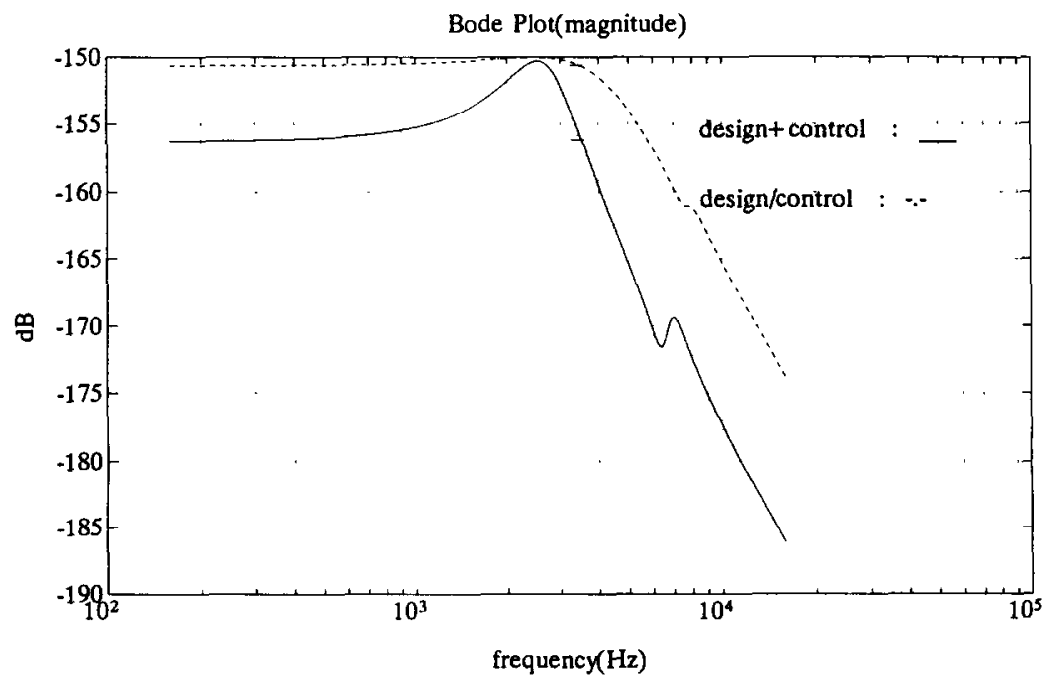

Fig. 8. Closed-loop frequency responses with goal programming designs $(\beta=1)$. 

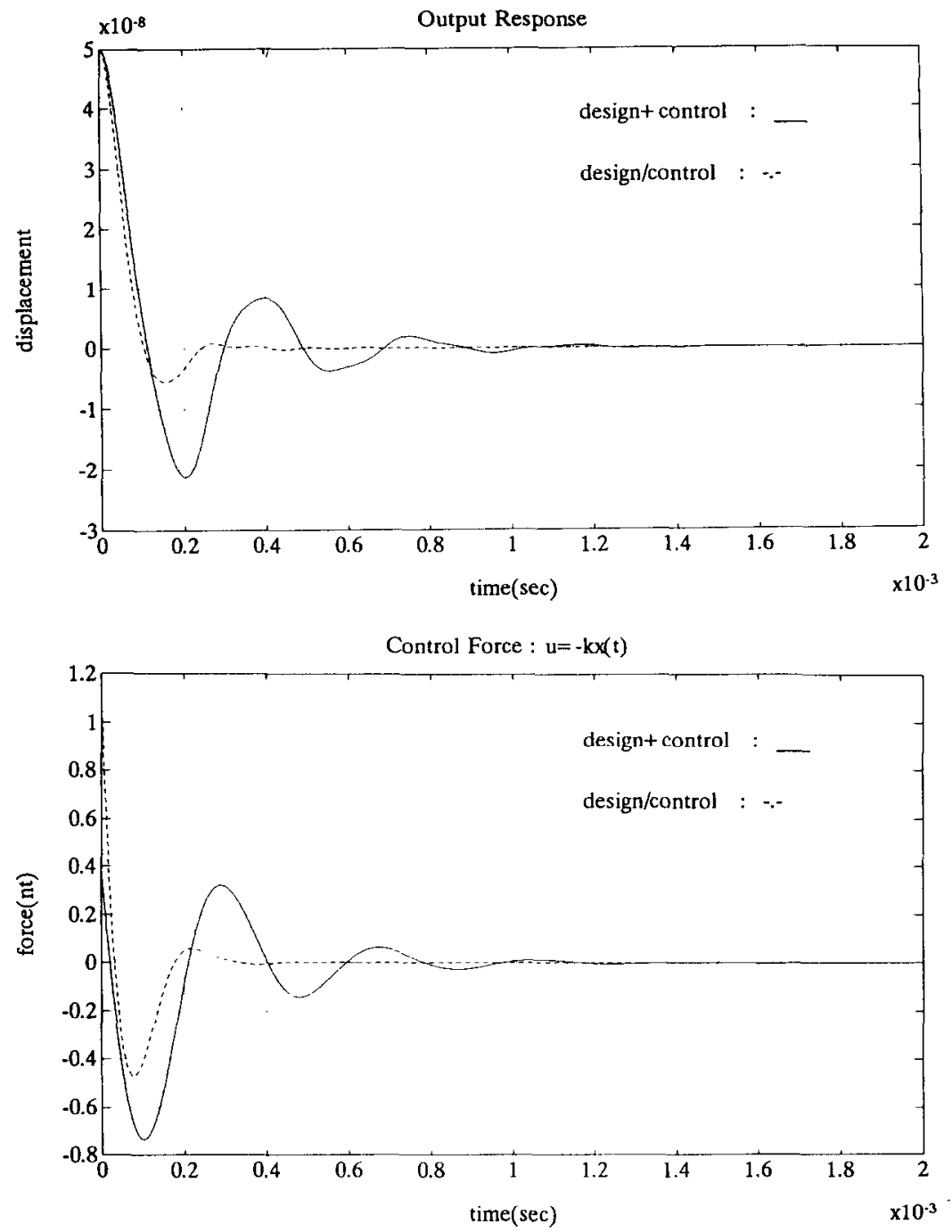

Fig. 9. Time responses with goal programming design $(\beta=1)$.

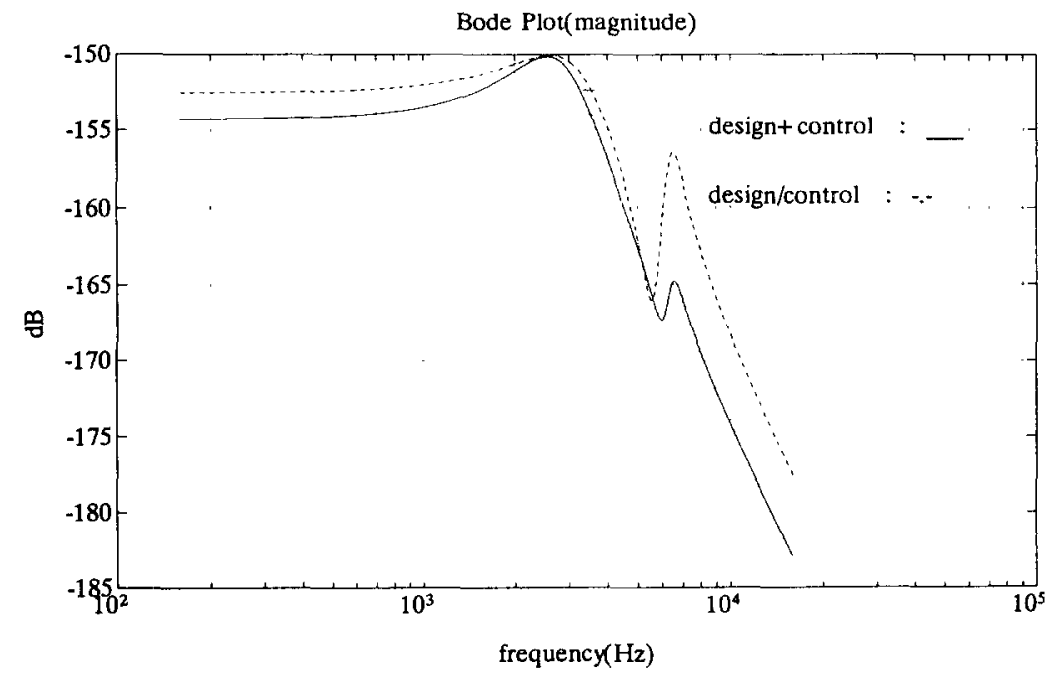

Fig. 10. Closed-loop frequency responses with compromise programming designs $(\gamma=1)$. 

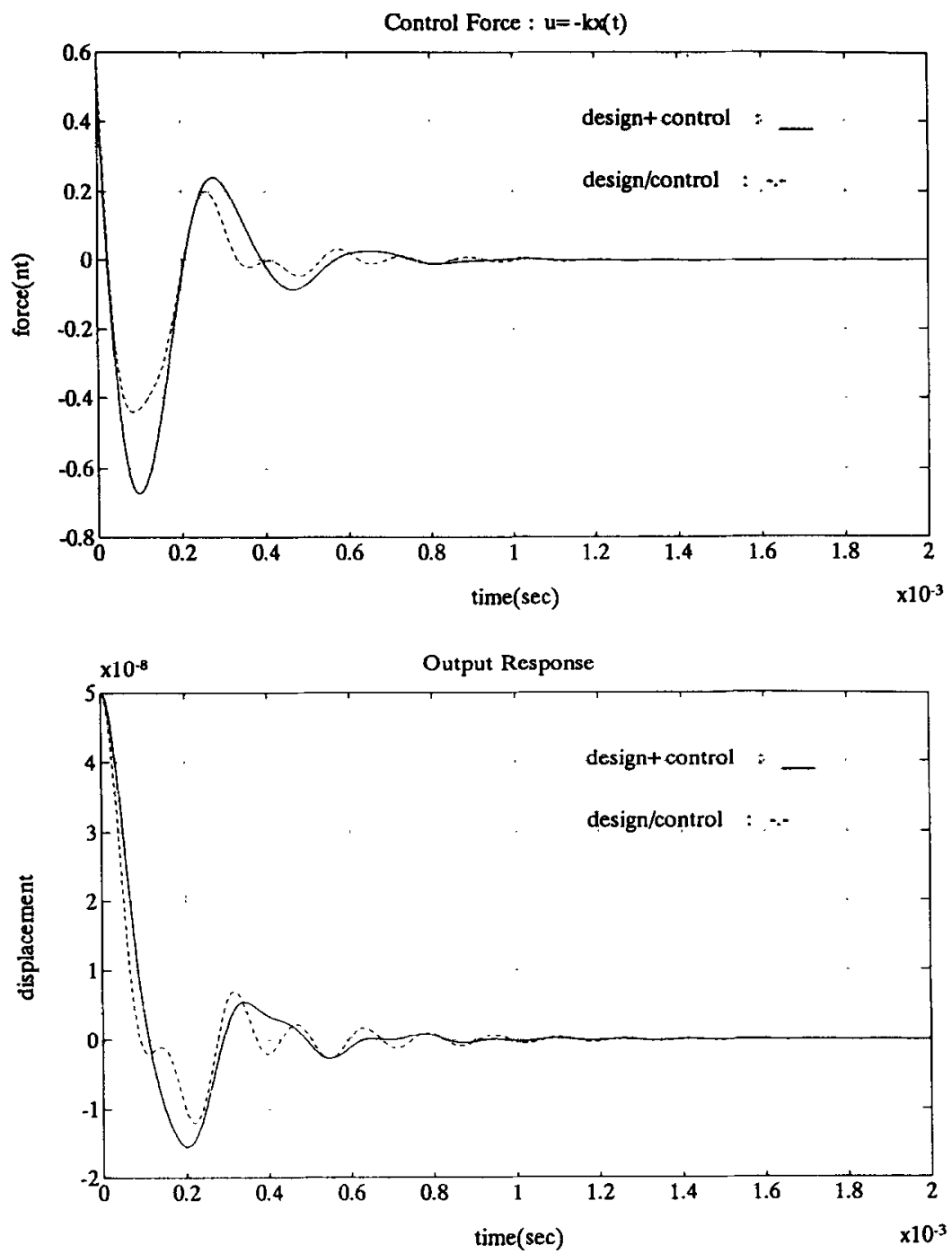

Fig. 11. Time responses with compromise programing design $(\gamma=1)$.

the slider has to be suppressed as soon as possible with moderate control effort. That way the goal programming design is the best desirable solution.

It is also necessary to convince people that the control performance of the suspension assembly designed with integrated structure/control optimization is superior to that without optimal control cost in the design as in Part I. Figure 8 is the frequency response of the suspension assembly with goal programming design. The solid curve (design + control) represents the case that the optimal control law is applied to the suspension designed with the objectives $f_{1}, f_{2}$ and $f_{3}$, while the dashdot curve (design/control) describes the closed-loop system frequency response with integrated optimization of structure and control. Both frequency responses have almost the same peak value and bandwidth, that is, the same frequency of input at which the output is attenuated to a factor 0.707 times the input. This is verified in Fig. 9 where both time responses have a similar rise time. However, the integrated structure/control design gives a much smaller resonant peak in frequency, which introduces more damping and leads to less settling time and less control effort in the regulation control. Similar results appear in the compromise programming design as shown in Figs 10 and 11.

\section{SUMMARY AND CONCLUSIONS}

The integrated structure/control multiobjective optimization of the suspension assembly of hard disk drives has been presented. In addition to the objective functions of natural frequencies, as depicted in Part I, also incorporated in the optimization is an optimal control performance index consisting of weighted system state regulation errors and control efforts. Two kinds of objectives have been achieved. First, the first and second natural frequencies are raised and the difference between the second and the third natural frequencies increases, so that the suspension is not 
easily excited by undesirable disturbances. Second, as a vibration controller is implemented it is required that the vibration be suppressed in minimal time and with least control effort. Both the goal programming and compromise programming techniques give feasible solutions, which would not be ideal, but the closest to the ideal ones in the sense that a best compromise is made among objectives. Furthermore, the find decision making requires more investigation on the closed-loop frequency and time responses. For the design of actively controlled structures, the control performances with the integrated structure/ control optimization design are superior to those without the optimal control performance index in the design. The final solution may not be unique, but depends on additional engineering experience, manufacturing requirements, and so on.

Acknowledgment--This research was supported by National Science Council under Contract no. NSC 82-0401-E-002-380.

\section{REFERENCES}

1. V. B. Venkayya and V. A. Tischler, Frequency control and its effect on the dynamic response of flexible structures. AIAA J. 23, 1768-1774 (1985).

2. R. V. Grandhi, Structural and control optimization of space structures. Comput. Struct. 31, 139-150 (1989).

3. J. Onoda and N. Watanabe, Integrated direct optimization of structure/regulator/observer for large flexible spacecraft. $A I A A J$. 28, 1677-1685 (1990).

4. G. L. Slater and M. D. McLaren, Disturbance model for control/structure optimization with full state feedback. J. Guid. Cotnrol Dyn. 16, 523-533 (1993).
5. D. S. Bodden and J. L. Junkins, Eigenvalue optimization algorithms for structure/controller design iterations. J. Guid. 8, 697-706 (1985).

6. A. L. Hale, R. J. Lisowski and W. E. Dahl, Optimal simultaneous structural and control design of maneuvering flexible spacecraft. J. Guid. 8, 86-93 (1985).

7. L. G. Horta, Jer-Nan Juang and J. L. Junkins, A sequential linear optimization approach for controller design. J. Guid. 9, 699-703 (1986).

8. D. F. Miller and J. Shim, Gradient-based combined structural and control optimization. J. Guid. 10, 291-298 (1987).

9. R. V. Lust and L. A. Schmit, Control-augmented structural synthesis. AIAA J. 26, 86-95 (1988).

10. H. L. Thomas, A. E. Sepulveda and L. A. Schmit, Improved approximations for control augmented structural synthesis. AIAA J. 30, 171-179 (1992).

11. C. H. Tseng and T. W. Lu, Multiobjective optimization in mechanical and structural design. Model. Sci. Comput. (submitted).

12. S. S. Rao, V. B. Venkayya and N. S. Khot, Game theory approach for the integrated design of structures and controls. AIAA J. 26, 463-469 (1988).

13. E. Livne, L. A. Schmit and P. P. Friedmann, Towards integrated multidisciplinary synthesis of actively controlled fibcr compositc wings. J. Aircraft 27, 979992 (1990).

14. M. G. Gilbert and D. K. Schmidt, Integrated structure/ control law design by multilevel optimization. $J$. Guid. 14, 1001-1006 (1991).

15. J. C. Bruch, S. Adali, J. M. Sloss and I. S. Sader, Optimal design and control of cross-ply laminate for maximum frequency and minimum dynamic response. Comput. Struct. 37, 87-94 (1990).

16. G. F. Franklin, J. D. Powell and A. Emami-Naeini, Feedback Control of Dynamic Systems, 3rd edn. Addison-Wesley, Reading, MA (1994). 